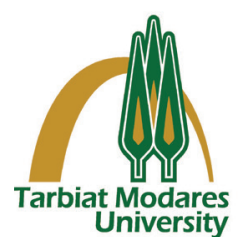

\title{
Molecular Detection of Cryptosporidium in Domestic Fowl in Shahrekord, Iran
}

\section{A R T I C L E I N F 0}

Article Type

Original Research

\section{Authors}

Maryam Karimi-Dehkordi, $P h D^{1}$ Majid Gholami-Ahangaran, $P h D^{1^{*}}$ Akram Ghahramani-Chermahini, $M S c^{2}$

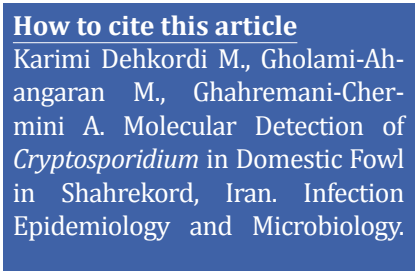

${ }^{1}$ Department of Clinical Sciences, Faculty of Veterinary Medicine, Shahrekord Branch, Islamic Azad University, Shahrekord, Iran. ${ }^{2}$ Department of Food Science and Technology, Ferdowsi University of Mashhad, Iran.

\section{* Correspondence}

Address: Department of Clinical Sciences, Faculty of Veterinary Medicine, Shahrekord Branch, Islamic Azad University, Shahrekord, Iran. Mgholami6@gmail.com

\section{Article History}

Received: October 05,2020

Accepted: December 25,2020

Published: January 23,2021

\begin{abstract}
A B S T R A C T
Background: Cryptosporidium is one of the most important pathogenic parasites in poultry because it is a zoonotic parasite, and birds and other infected animals could be a potential threat to public health. The main purpose of this study was to determine the frequency of Cryptosporidium infection in domestic fowl in Shahrekord by PCR method.

Materials \& Methods: In this cross-sectional study, 110 fecal samples were collected from fowls referred to the Veterinary Clinic of Islamic Azad University, Shahrekord Branch. After DNA extraction, the samples were examined by PCR, and the frequency of infection in different genders and seasons was analyzed by SPSS statistical software.

Findings: Out of 110 samples, \%13.64) 15) samples were positive for Cryptosporidium. The rate of Cryptosporidium infection in the females was $\% 12.85$ and in the males was \%15. The results also showed that there was no statistically significant difference between two sexes (male and female) regarding the prevalence of Cryptosporidium, while the frequency of infection in cold seasons (\%22.22) was significantly higher than in warm seasons (\%7.69). Conclusion: Fowls could be considered as one of the important reservoirs of Cryptosporidium infection for humans.
\end{abstract}

\section{Keywords: Cryptosporidium, Fowl, Zoonotic, PCR.}

\section{CITATION LINKS}

[1] Kabir MH, Han Y, Lee SH, Nugraha AB, Recuenco F, Murakoshi F, et al. Prevalence and molecular characterization of Cryptosporidium species in poultry in ... [2] Helmy YA, Krücken J, Abdelwhab ES, von Samson-Himmelstjerna G, Hafez HM. Molecular diagnosis and characterization of Cryptosporidium spp. in turkeys and chickens in Germany reveals evidence for previously... [3] Nguyen TT, Traub R, Pham PD, Nguyen HV, Nguyen KC, Phung $\mathrm{CD}$, et al. Prevalence and molecular characterization of Cryptosporidum spp. and Giardia spp. in environmental samples in Hanam ... [4] FAO W. Multicriteria-based ranking for risk management of foodborne parasites. Report of a joint FAO/WHO expert meeting. Rome: Food ... [5] Hashemzadeh F, Shahbazi P, Bahavarnia SR. Investigation of ... [6] Jordan F. Poultry disease. 6th ... [7] Atyabi N, Poursafar F, Hesam S, Hashemian M. Evaluation of Cryptosporidium in ... [8] Wang L, Xue X, Li J, Zhou Q, Yu Y, Du A. Cryptosporidiosis in broiler chickensinZhejiang province, China: Molecular... [9] Ghasemian O, Youssefi M.R, Hossienzadeh Marzenaki J. Prevalence and ... [10] Nili H, Asasi K. Natural cases and an... [11] de Graaf DC, Vanopdenbosch E, Ortega-Mora LM, Abbassi H, Peeters JE. A review of... [12] Banani M, Dadras H, Moazeni JG, Houshmandrad P. Serologic ... [13] Baroudi D, Khelef D, Goucem R, Adjou KT, Adamu H, Zhang H, et al. Common occurrence of zoonotic pathogen Cryptosporidium meleagridis in broiler chickens and turkeys in... [14] Kuzehkanan AB, Rezaeian M, Zeraati H, Mohebali M, Meamar AR, Babaei Z, et al. A sensitive and specific PCR based method for identification of Cryptosporidium spp. using new primers from 18S ribosomal RNA. Iran J ... [15] Soltanialvar M, Razijalali MH. Prevalence of Cryptosporidium parasite infection in turkeys in Dezful, J Vet ... [16] Checkley W, White Jr AC, Jaganath D, Arrowood MJ, Chalmers RM, Chen XM, et al. A review of... . [17] Goodwin MA. Cryptosporidiosis in... [18] Jenkins M, O`Brien C, Fetterer R, Santin M. RT-PCR specific for Cryspovirus is a highly sensitive method for... [19] Xiao L, Escalante L, Yang C, Sulaiman I, Escalante AA, Mantali RJ, et al. ... [20] Ahmadi-Gharacheh M, Gholami-Ahangaran M, Momtaz H. Molecular detection of Cryptosporidium as ... [21] Heydari H, Gharakhani J. Study of Cryptosporidium... [22] Nouri M, Bozorgmehri M, Mansouri N, Evaluation of respiratory and... [23] Haghbin Nazarpak H, Mousavi SA, RanjbarBahadori Sh, ... [24] Wang R, Jian F, Sun Y, Hu Q, Zhu J, Wang F, et al. Large-scale ... [25] Goodwin MA, Brown J. Histology... [26] Esteban JG, Aguirre C, Flores A, Strauss W, Angles R, Mas-Coma S. High... [27] Clavel A, Olivares JL, Fleta J, Castillo J, Varea M, Ramos FJ, et al. Seasonally ... [28] Mata L, Bolaños H, Pizarro D, Vives M. Cryptosporidiosis in children from... [29] Tzipori S, Smith M, Birch C, Barnes G, Bishop R. Cryptosporidiosis in hospital patients with... . 


\section{Introduction}

Cryptosporidium is a coccidian protozoa with a notable reproduction and proliferation capacity. It is extensively found in wild and domestic animals species around the world. The parasite is one of the most prevalent parasites in poultry so that it could be found in 30 species of birds worldwide such as chicken, turkey, duck, geese, quail, pheasant, and peacock ${ }^{[1-3]}$. According to the specialized committee of FAO/WHO, Cryptosporidium is the fifth top cause of zoonosis, which could be transferred through food ${ }^{4]}$. Throughout its growth process, the parasite could be found in the respiratory, digestive, and urinary systems of birds. The produced oocyte by the parasite causes respiratory and digestive infections. The major symptom of Cryptosporidium infection is continuous diarrhea ${ }^{[5]}$. The respiratory system infection is more severe than that of other organs and causes symptoms like nose secretions, respiratory sign, and pneumonia. Digestive infection in the host bird is caused by benign Cryptosporidium so that the parasite damages the intestine tissue and causes mild diarrhea ${ }^{[6-8]}$. Cryptosporidium disrupts the function and physiology of the intestine and causes pathological damages to the intestine cells, leading to other intestinal and digestive complications ${ }^{[9]}$. Moreover, the disease could be lethal or delay the growth process in poultry. In addition, the infected birds become susceptible to secondary infections, especially respiratory infections [10]. While Cryptosporidium causes diseases in a wide range of animals, it was neglected until the early 1980s. Since then, the parasite has been found as one of the serious and common causes of diarrhea in some mammals and birds species. Investigation of the relationship between Cryptosporidium and the prevalence of diarrhea with aquatic source in humans led to a wider knowledge about the parasite [11]. Cryptosporidium contaminates soil and water through feces, oral secretions, and eggs that stay alive in the environment. Therefore, it has several ways to enter the food chain ${ }^{[12]}$. Oocytes are considered as a potential source of infection in humans so that infection could occur through consuming contaminated food and water or direct contact with contaminated individuals or animals. There are several cases of the parasite infection in individuals, caused by direct contact with animals and poultry [13]. It has been found that Cryptosporidium could cause clinical and subclinical respiratory, digestive, urinary, and genital diseases in human. The parasite may cause even life-threatening infections in individuals with immunodeficiency, such as HIV infected persons [14-15]. Therefore, the parasite is important in terms of public health. As noted earlier, Cryptosporidium could cause common diseases in many animals such as cow, sheep, goat, dog, cat, horse, poultry, and turkey. Due to zoonotic importance of this protozoa and the risk of human food and water contamination and higher risk of transmission to human, it is important to measure the prevalence and infection load of the parasite and introduce efficient control and prevention policies [1617]. Preventing the spread of Cryptosporidium in environmental samples needs advanced and accurate methods. In addition, zoonotic pandemics could be prevented through a proper management of the parasite sources [18].

Objectives: Given what was said in the introduction, the paucity of studies on the prevalence of Cryptosporidium, and the presence of a large number of poultry farms in Chaharmahal-va-Bakhtiari, Iran, the present study was an attempt to examine the frequency of the parasite in domestic poultry in Chaharmahal-va-Bakhtiari through PCR method. By having accurate information about the prevalence of the parasite, better 
preventive and health programs could be provided to prevent further spread of the parasite in poultry and its transmission to humans.

\section{Materials and Methods}

Sampling: To examine the frequency of Cryptosporidium in domestic poultry, a crosssectional study was carried out in a one-year period on 110 feces samples brought to the Veterinary Clinic of Islamic Azad University, Shahrekord Branch. Sampling was done using a sterile swab from cloaca region. The samples were kept at $-70{ }^{\circ} \mathrm{C}$ after sampling until use for further examination.

DNA extraction: Phenol-chloroform method and standard procedures were used for DNA extraction (SinaClon, Irans).

PCR: The primers used for the detection of Cryptosporidium were synthesized according to Xiao et al. (1999) ${ }^{[19]}$. The characteristics of the primers used are presented in Table 1. The PCR conditions were optimized according to Ahmadi-Gharacheh et al. (2020) Table 1) Sequence of the primers used to amplify ssrRNA of Cryptosporidium ${ }^{[19]}$

\begin{tabular}{llc}
\hline Band Length (bp) & Primer Sequence (5' to 3') & Product (bp) \\
\hline ssrRNA-F & TTCTAGAGCTAATACATGCG & 1325 \\
SsrRNS-R & CCCATTTCCTTCGAAACAGGA & \\
\hline
\end{tabular}

Table 2) Total percentage of Cryptosporidium infection in both sexes of domestic fowls in different seasons

\begin{tabular}{|c|c|c|c|c|c|c|c|c|c|}
\hline \multirow[b]{2}{*}{ Seasons } & \multicolumn{3}{|c|}{ Female } & \multicolumn{3}{|c|}{ Male } & \multicolumn{3}{|c|}{ Total } \\
\hline & Total No. & Positive No. & $(\%)$ & Total No. & Positive No. & $\%$ & Total No. & Positive No. & $\%$ \\
\hline Summer & 28 & 2 & 7.14 & 12 & 1 & 8.33 & 40 & 3 & 7.5 \\
\hline Fall & 15 & 2 & 13.33 & 10 & 1 & 10 & 25 & 3 & 12 \\
\hline Winter & 12 & 4 & 33.33 & 8 & 3 & 37.5 & 20 & 7 & 35 \\
\hline Spring & 15 & 1 & 6.66 & 10 & 1 & 10 & 25 & 2 & 8 \\
\hline Total & 70 & 9 & 12.85 & 40 & 6 & 15 & 110 & 15 & 13.64 \\
\hline
\end{tabular}


illustrates the electrophoresis results.

Totally, 110 specimens were examined by PCR method, of which 15 samples were found to be positive (13.64\%) (Table 2).

As the results showed, the frequency of Cryptosporidium in male fowls (six out of 40; $15 \%)$ was slightly higher than in female fowls (nine out of $70 ; 12.85 \%$ ). Based on the Chisquare test results, there was no significant relationship between the frequency of Cryptosporidium and gender (male/female) (Chi-square value $=0.0104 ; p=.918)$ (Table 3$)$.

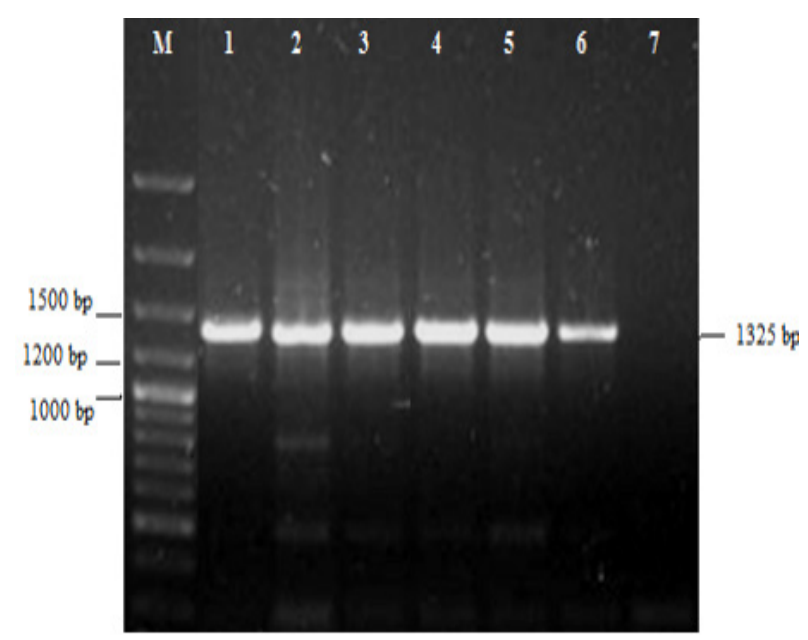

Figure 1) Electrophoresis of PCR product of ss rRNA gene of Cryptosporidium. (column M: marker; column 1-6: positive samples; and column 7: negative sample)

Table 3) Cryptosporidium infection in domestic hens and cook.

\begin{tabular}{lcc}
\hline Gender & Positive & Negative \\
\hline Female & 9 & 61 \\
Male & 6 & 34 \\
\hline
\end{tabular}

The frequency distribution analysis in cold and hot seasons showed that the frequency of Cryptosporidium was higher in cold seasons. Clearly, the highest frequency was observed in winter (seven out of 20;35\%), and the lowest frequency was observed in summer (three out of $40 ; 7.5 \%)$. There was a significant difference between warm and cold seasons in terms of the positive cases of Cryptosporidium (Chisquare value $=4.767 ; p=.029$ ) (Table 4).

Table 4) Cryptosporidium infection in domestic fowls in cold and warm seasons

\begin{tabular}{lcc}
\hline Season & Positive & Negative \\
\hline $\begin{array}{l}\text { Cold seasons (fall } \\
\text { and winter) }\end{array}$ & 10 & 35 \\
$\begin{array}{l}\text { Hot seasons (spring } \\
\text { and summer) }\end{array}$ & 5 & 60 \\
\hline
\end{tabular}

\section{Discussion}

Cryptosporidium is an intracellular protozoa parasite of the digestive, respiratory, and urinary systems of vertebrates. It is one of the zoonotic factors that could impose several risks to the health of poultry, livestock, and human. There are several reports indicating an increase in the prevalence of Cryptosporidium in developing countries, which highlight the importance of the parasite in terms of public health. The main ways of transferring the parasite are direct contact or exposure to contaminated water and soil. Given the growing trend of the parasite prevalence and in turn contaminated sites, the parasite has become an environmental concern as well. The prevalence of the parasite, like other zoonotic diseases, is not the same in different regions or countries due to different management systems, geographical conditions, climates, population of animals, susceptibility to the parasite, contact between different species, and etiological specifications. Studies have shown that the parasite is highly prevalent in most countries worldwide. The parasite prevalence in industrial poultry herds ranges from 1 to $50 \%{ }^{[21]}$. There are a few studies in Iran, investigating the prevalence and frequency of Cryptosporidium in poultry based on molecular identification. Most studies on this topic in Iran have been based on clinical and microscopic observations. The present study examined 
Cryptosporidium infection in domestic fowl in Shahrekord using PCR method and reported the frequency of this infection as 13.64\%. There are similar studies in Iran and other countries, which have investigated the prevalence of Cryptosporidium, many of which have reported similar results with the present work. Hashemzadeh et al. (2014) studied broiler chickens and found 36 positive cases out of 400 samples (9\%). In addition, the disease prevalence rate was higher in winter (15\%), which was significantly different from other seasons based on Chi-squared test. In addition, out of 400 samples, 290 were female with 27 positive cases, and 110 were male with nine positive cases. They found no significant difference between two genders in terms of the parasite prevalence ${ }^{[5]}$, which is in line with the present study result. Banani et al. (2000) assessed the serologic prevalence of Cryptosporidium in broiler flocks in Shiraz using ELISA method. Out of 1522 serum samples of 26 broiler flocks, 125 were positive ${ }^{[12]}$. Haghbin et al. (2021) examined 300 samples collected from 30 broiler farms using microscopic method. They reported that seven farms (23.33\%) were contaminated with the parasite, and 39 samples (13\%) were positive ${ }^{[23]}$. According to Nouri et al. (1994), the prevalence rate of Cryptosporidium in the feces samples collected from industrial broiler farms around Tehran was $2.25 \%{ }^{[22]}$. The prevalence rate reported by these studies is less than that reported in the present study. It is worth noting that domestic poultry are kept in open spaces, and there is no control on their feed. In addition, domestic poultry are in contact with other wild birds, thereby increasing the prevalence of Cryptosporidium compared to poultry in industrial systems. Studies in other countries have reported different prevalence rates. A study by Helmy et al. (2017) on 256 feces samples randomly collected from poultry in industrial systems in Germany examined infectivity caused by different Cryptosporidium species using PCR method. They found 18 positive chickens, of which nine chickens belonged to broilers. Their study was the first work in Germany using molecular methods to find Cryptosporidium in poultry. It was found that Cryptosporidium was prevalent in broiler chickens and turkeys in Germany ${ }^{[2]}$. A study by Wang et al. (2014) showed that out of 385 broiler chickens collected from seven regions in China, 38 chickens were positive $(10 \%)^{[8]}$. Baroudi et al. (2013) examined 90 dead chickens collected from 23 farms and 57 dead turkeys from 16 farms in Algeria using PCR-RFLP method on a small rRNA gene. They reported 34 and $44 \%$ infectivity due to Cryptosporidium in chicken and turkey samples, respectively ${ }^{[13]}$. Another study by Wang et al. (2010) on ducks and chickens in China reported the prevalence rate of Cryptosporidium as $10.6 \%$ (163 out of 1542). The prevalence rate in broiler chickens was reported to be $3.4 \%$. The highest prevalence rate was observed in spring (15.6\%), and the lowest prevalence rate was observed in winter ${ }^{[24]}$, which is different from the present study findings. A similar study in the USA showed that the prevalence rate in spring (24 out of 267) was the highest compared to other seasons, and the lowest prevalence rate was in winter (nine out of 260) so that there was a significant difference between seasons ${ }^{[25]}$. The present study showed that the highest prevalence rate was in winter, which is inconsistent with the aforementioned studies results. One reason for this could be the small sample size and study population. Several studies have mentioned environmental and socio-economic condition as the factors influencing the seasonal prevalence of Cryptosporidium ${ }^{[26-29]}$. Temperature, rainfall, humidity, farming method, and contact 
with other animals are among the probable factors affecting the seasonal prevalence of the parasite [27]. The present study showed that the frequency of Cryptosporidium was higher in cold seasons, which could be due to higher survival rate of the parasite in moist condition.

\section{Conclusion}

According to the results of this and aforementioned studies, the frequency of Cryptosporidium in domestic fowls in Shahrekord-Iran and the suburbs was high. Apparently, domestic fowls could be a source of Cryptosporidium infection that endangers the health of human population. Given the close contact between poultry and the local population, it is essential to provide education and awareness to the local population and those interested in keeping domestic animals. In addition, given the popularity of keeping domestic animals in the region and the growing number of industrial poultry farms in the province, more comprehensive studies on the prevalence of Cryptosporidium in the region are required to be performed.

Acknowledgments: The authors would like to appreciate all persons that help them in this research, comprising owners of fowls and under-graduated students in Faculty of Veterinary Medicine, Islamic Azad University, Shahrekord Branch, Iran.

Ethical Permission: This study was confirmed and approved by Ethics committee of Sharekord Branch, Islamic Azad university.

Authors Contribution: Conceptualization: MGA \& MKD; Data curation and formal analysis: MGA \& MKD; Investigation: MKD \& MGA; Methodology and project administration: AGC \& MHS; Supervision: MGA \& MKD; Validation: MHS \& MKD; Writing of original draft: MKD; Writing, reviewing, and editing: MGA \& AGC.
Conflicts of Interests: The authors declared no conflict of interest.

Funding: Self-funding.

Consent to participate: Consent was obtained from each fowl owner.

\section{References}

1. Kabir MH, Han Y, Lee SH, Nugraha AB, Recuenco F, Murakoshi F, et al. Prevalence and molecular characterization of Cryptosporidium species in poultry in Bangladesh. One Health. 9:100122;2020.

2. Helmy YA, Krücken J, Abdelwhab ES, von Samson-Himmelstjerna G, Hafez HM. Molecular diagnosis and characterization of Cryptosporidium spp. in turkeys and chickens in Germany reveals evidence for previously undetected parasite species. PloS One. 6)12;2017):e0177150.

3. Nguyen TT, Traub R, Pham PD, Nguyen HV, Nguyen KC, Phung CD, et al. Prevalence and molecular characterization of Cryptosporidum spp. and Giardia spp. in environmental samples in Hanam province, Vietnam. Food Waterborne Parasitol. 20-3:13;2016.

4. FAO W. Multicriteria-based ranking for risk management of foodborne parasites. Report of a joint FAO/WHO expert meeting. Rome: Food and Agriculture Organization; 2014.

5. Hashemzadeh F, Shahbazi P, Bahavarnia SR. Investigation of Cryptosporidium parasite infection in native poultry around Tabriz. JVCP. 95-588:(3)8;2014.

6. Jordan F. Poultry disease. 6th ed. US: Saunders Ltd; 2008.

7. Atyabi N,Poursafar F, Hesam S, Hashemian M. Evaluation of Cryptosporidium in feces and oral-pharyngeal secretions of ducks and poultry in Gilan and Golestan provinces.

8. Wang L, Xue X, Li J, Zhou Q, Yu Y, Du A. Cryptosporidiosis in broiler chickens in Zhejiang province, China: Molecular 
characterization of oocysts detected in fecal samples. Parasite. 42-21:36;2014.

9. Ghasemian O, Youssefi M.R, Hossienzadeh Marzenaki J. Prevalence and distribution of Eimeria species and Cryptosporidium infection in indigenous fowls of Behbahan, south west Iran. Vet Microbiol. 62-57:(2)1;2019.

10. Nili H, Asasi K. Natural cases and an experimental study of H9N2 avian influenza in commercial broiler chickens of Iran. Avian Pathol. 52-31:247;2002.

11. de Graaf DC, Vanopdenbosch E, OrtegaMora LM, Abbassi H, Peeters JE. A review of the importance of cryptosporidiosis in farm animals. Int J Parasitol. 87-1269:(8)29;1999.

12. Banani M, Dadras H, Moazeni JG, Houshmandrad P. Serologic incidence of cryptosporidial infection in broiler flocks in Shiraz, Iran. Arch Razi Ins. 102-51:95;2000.

13. Baroudi D, Khelef D, Goucem R, Adjou $\mathrm{KT}$, Adamu $\mathrm{H}$, Zhang $\mathrm{H}$, et al. Common occurrence of zoonotic pathogen Cryptosporidium meleagridis in broiler chickens and turkeys in Algeria. Vet Parasitol. 40-334:(4-3)196;2013.

14. Kuzehkanan AB, Rezaeian M, Zeraati $H$, Mohebali M, Meamar AR, Babaei Z, et al. A sensitive and specific PCR based method for identification of Cryptosporidium spp. using new primers from $18 \mathrm{~S}$ ribosomal RNA. Iran J Parasitol. 8-1:(4)6;2011.

15. Soltanialvar M, Razijalali MH. Prevalence of Cryptosporidium parasite infection in turkeys in Dezful, J Vet Histol. $10-100:(3) 2 ; 2015$.

16. Checkley W, White Jr AC, Jaganath D, Arrowood MJ, Chalmers RM, Chen XM, et al. A review of the global burden, novel diagnostics, therapeutics, and vaccine targets for Cryptosporidium. Lancet Infect Dis. 94-85:(1)15;2015.

17. Goodwin MA. Cryptosporidiosis in birds-A review. Avian Pathol. 84-365:(3)18;1989.
18. Jenkins M, O〉Brien C, Fetterer R, Santin M. RT-PCR specific for Cryspovirus is a highly sensitive method for detecting Cryptosporidium parvum oocysts. Food Waterborne Parasitol. 20-5:14;2016.

19. Xiao L, Escalante L, Yang C, Sulaiman I, Escalante AA, Mantali RJ, et al. Phylogenetic analysis of Cryptosporidium parasites based on the small subunit rRNA gene locus. Appl Environ Microbiol. 83-1578:(4)65;1999.

20. Ahmadi-Gharacheh M, GholamiAhangaran M, Momtaz H. Molecular detection of Cryptosporidium as a zoonotic pathogen, in pet birds of Isfahan, Iran. J Gorgan Univ Med Sci. $103-99$ : (2)22;2020.

21. Heydari H, Gharakhani J. Study of Cryptosporidium infection in the livestock (cattle, sheep, dogs, fowls) and humans in Hamadan city and its suburbs during the years 2011-2006. Avicenna J Clin Med. 74-67:(3)19;2012.

22. Nouri M, Bozorgmehri M, Mansouri $\mathrm{N}$, Evaluation of respiratory and gastrointestinal cryptosporidiosis in industrial poultry in Tehran. J Vet Res. 6-100:(2)49;1994.

23. Haghbin Nazarpak H, Mousavi SA, RanjbarBahadori Sh, Mohammadi Malayeri MR, Hoseini SM. Frequency of Cryptosporidium infection in broiler breeding flock of Ghaemshahr. J Vet Microbiol. 5-1:(1)7;2011.

24. Wang R, Jian F, Sun Y, Hu Q Zhu J, Wang F, et al. Large-scale survey of Cryptosporidium spp. in chickens and Pekin ducks (Anas platyrhynchos) in Henan, China: Prevalence and molecular characterization. Avian Pathol. 51 -447:(6)39;2010.

25. Goodwin MA, BrownJ.Histology incidence and distribution of Cryptosporidium spp infection in chickens. J AmVet Med Assoc. 1623-1623;(12)190;1987. 
26. Esteban JG, Aguirre C, Flores A, Strauss W, Angles R, Mas-Coma S. High Cryptosporidium prevalences in healthy Aymara children from the northern Bolivian Altiplano. Am J Trop Med Hygiene. 5-50:(1)58;1998.

27. Clavel A, Olivares JL, Fleta J, Castillo J, Varea M, Ramos FJ, et al. Seasonally of cryptosporidiosis in children. Eur J Clin Microbiol Infect Dis. 9-77:(1)15;1996.
28. Mata L, Bolaños H, Pizarro D, Vives M. Cryptosporidiosis in children from some highland Costa Rican rural and urban areas. Am J Trop Med Hygiene. 9-24:(1)33;1984.

29. Tzipori S, Smith M, Birch C, Barnes G, Bishop R. Cryptosporidiosis in hospital patients with gastroenteritis. Am J Trop Med Hygiene. 4-931:(5)32;1983. 